\title{
Genre et Communication : quels enjeux pour les pratiques professionnelles en communication?
}

Valérie Lépine, professeure des universités en Sciences de l'information - communication à l'ITIC, Université Paul Valéry - Montpellier 3, Membre du LERASS valerie.lepine@univ-montp3.fr

Josianne Millette, professeure agrégée Département d'information et de communication de l'Université Laval (Québec, QC), membre du Laboratoire de communication médiatisée par ordinateur (LabCMO).

josianne.millette@com.ulaval.ca

Sandrine Roginsky, professeure, École de communication de l'UCLouvain, directrice du LASCO,

membre associée du Laboratoire de communication médiatisée par ordinateur

(LabCMO)

sandrine.roginsky@uclouvain.be 
Le genre apparaît comme une dimension non négligeable dans l'appréhension des métiers et des parcours professionnels de la communication dans toutes ses déclinaisons (relations publiques, presse, publicité, journalisme d'entreprise, communication externe, interne, etc.). On trouve en sociologie du travail des recherches s'intéressant au thème de la féminisation des métiers et des professions, qui, par exemple, interrogent une possible dévalorisation induite par l'arrivée des femmes, se penchent sur une division du travail « où se révèlent les principes de différenciation et de hiérarchisation entre les tâches masculines et féminines » (Malochet, 2007, § 6), ou encore posent la question des discriminations en organisations. Ces travaux amènent à considérer la transversalité des rapports de genre, puisque les trajectoires professionnelles doivent être articulées aux modalités de travail et aux activités proprement dites. D'ailleurs, pour Miller (cité par Caron, 2004), le genre occupe une position centrale dans la vie organisationnelle.

Ainsi, depuis quelques années, certaines associations professionnelles, notamment COM-ENT en France, ont pris l'initiative d'animer des réflexions sur les pratiques professionnelles et d'engagement contre le sexisme (« Toutes Femmes, Toutes Communicantes »). Un mouvement similaire s'observe en Belgique, avec notamment une réflexion des communicateurs et communicatrices des institutions publiques sur la dimension genrée de la communication produite, ainsi qu'au Canada et ailleurs dans le monde, avec, par exemple, la mise sur pied de regroupements et campagnes comme «Femme en Créa» (Québec), The Lost Girls Mouvement (EU) et Creative Equals (UK). La mise en place de standards et de guides pour communiquer en évitant de mobiliser des stéréotypes de genre et en favorisant différentes formes d'écriture inclusive s'est également répandue dans de nombreuses institutions publiques et éducatives. Les qualificatifs varient cependant; définir le problème ne fait pas consensus, comme d'ailleurs ne fait pas consensus le type d'écriture à mobiliser pour prendre en compte la dimension genrée de la communication. Enjeu de communication, enjeu pour les communicateurs et communicatrices, la question du genre est donc centrale. Pour Bertini (2006, 121) d'ailleurs, « toute situation d'information et de communication se réfère implicitement au système de signification et d'interprétation que constitue le genre », tandis que Coulomb-Gully $(2009, \S 32)$ estime que « toute communication est par définition genrée ».

Pourtant, la question du genre peut paraître délaissée par les sciences de l'information et de la communication. Les questions relatives au rapport entre genre et pratiques professionnelles des communications ont été davantage investies dans la littérature anglo-saxonne, en relations publiques notamment (Daymon et Demetrious, 2014). Les enjeux de la féminisation y trouvent une forte résonance, comme peut d'ailleurs l'illustrer la récente étude menée par EUPRERA qui propose une revue de littérature extensive, de 1982 à 2019 et met en évidence les enjeux de genre auxquels les femmes qui pratiquent le métier sont confrontées (Topic et al., 2019). Par contraste, en France, Virginie Julliard constate qu' « aucun courant de recherche ne s'est constitué autour de la problématique du genre et de la communication »(Julliard, 2009, § 9). Les 
travaux engagés en SIC au prisme du genre demeurent dans une relative discrétion (Fleury et Walter, 2009) même si une certaine visibilité leur a été donnée grâce au dossier que la Revue française des sciences de l'information et de la communication (RFSIC) a consacré en 2014 aux « recherches au féminin en SIC ». Dans le dossier qu'elles ont coordonné, Françoise Bernard et Catherine Loneux ont voulu, d'une part, présenter des recherches qui portent sur le genre en communication et, d'autre part, souligner la contribution des femmes à la recherche en SIC produisant des analyses et des publications structurantes dans tous les domaines de la recherche en SIC, indépendamment des Gender Studies (Bernard et Loneux, 2014).

Quand la question du genre est abordée, elle l'est essentiellement par le biais de l'analyse des représentations dans la publicité ou les médias et, dans une moindre mesure, dans le domaine de la communication politique - notamment sous l'effet des politiques publiques en faveur de la parité - avec par exemple un intérêt pour l'analyse des régimes discursifs relatifs au genre (Julliard, 2009). Au Québec, si le même écart s'observe entre productions anglo-saxonne et francophone sur le genre en études de l'information et de la communication, ces deux littératures sont de moins en moins cloisonnées. Par ailleurs, les chercheures et chercheurs francophones du Québec participent activement à l'actualisation des perspectives culturelles sur le genre et les médias en français, à l'instar de leurs collègues franco-européens (Coulomb-Gully, 2010). Les connaissances concernent cependant surtout le journalisme, les contenus de fiction ou, là aussi, la représentation des personnalités politiques; les pratiques de marketing ou de relations publiques sont encore peu explorées au prisme du genre. Ainsi, dans l'ensemble, ces recherches portent, comme le rappelle Coulomb-Gully (2009), davantage sur les médias que sur la « communication » ou « l'informationcommunication ». Si l'histoire du genre et des médias, comme les questionnements intersectionnels en communication, prennent par ailleurs progressivement de l'importance, c'est partout l'étude des représentations genrées qui domine, alors que les dynamiques de production et de réception demeurent marginales comme objet d'étude.

En effet, le genre n'est encore que rarement objet ou prisme de recherche quand la communication est étudiée sous l'angle de la professionnalisation de ses pratiques et de ses acteurs. Ce, alors même qu'il a été documenté que les études de communication sont généralement plébiscitées par les femmes (Baudelot, Establet, in Blöss, 2011, cités par Coulomb-Gully, 2009) et que certains secteurs professionnels, comme celui des relations publiques, sont parfois qualifiés de « secteur genré » (" gendered field») (Aldoory et Toth, 2002). Présente dès les filières de formation, la « surreprésentation » actuelle des femmes dans la communication doit cependant être analysée finement dans le contexte des différents métiers et des postes qui y sont occupés. En France par exemple, les femmes sont majoritaires à enseigner dans ce champ académique : $54 \%$ des enseignant es-chercheur es en SIC sont des femmes mais elles ne sont que 
$44 \%$ parmi les professeur·es d'universités (poste le plus élevé) en 2019-20201 - elles sont moins de $30 \%$ au plus haut niveau si l'on compte toutes les disciplines. Les sociologues du monde professionnel qui s'intéressent aux problématiques de genre soulignent en effet, dans de nombreux domaines, une double ségrégation verticale et horizontale à laquelle le secteur de l'information-communication n'échappe pas (Neveu, 2000). Dès les années 1990, ces réalités ont fait l'objet de travaux dans la littérature anglo-saxonne en relations publiques, inaugurant une série de recherches qui se sont intéressées à ce « plafond de verre » et, plus généralement, aux assignations différenciées des rôles et des statuts au sein de ce corps de métier (Fitch et Third, 2014 ; Grunig, Toth et Hon, 2001 ; Toth et Grunig, 1993). Or, comme Manon Niquette (2000) l'a montré, cette problématisation - s'inscrivant dans une approche libérale - de la « féminisation » de la profession relève d'une invisibilisation des femmes, présentes en réalité tout au long de l'histoire de la professionnalisation des relations publiques et de la communication, et à laquelle tentent de répondre des travaux retraçant une « histoire au féminin » (herstory) de la pratique (Fitch, 2020). D'autres travaux ont également attiré l'attention sur la façon dont les milieux professionnels tendent à valoriser certains critères de féminité ou de masculinité, ce qui peut se refléter tant à l'embauche que dans le type de postes occupés (Elmer, 2010; Tindall et Waters, 2012).

Pourtant, nous ne trouvons pas de trace significative de travaux en SIC s'intéressant par exemple à ce que le genre fait aux pratiques professionnelles - des femmes mais également des hommes - en communication, alors même que le genre offre l'occasion « de renouveler les approches en articulant cette nouvelle problématique à d'autres déjà reconnues » (Julliard, 2009). Levier d'interrogation, le genre conduit ainsi à faire émerger des questionnements nouveaux quant à la professionnalisation et aux métiers de la communication.

L'appel à contribution à l'origine de ce dossier souhaitait ainsi ouvrir un espace de discussion et de réflexion, autant pour les chercheures et chercheurs que les membres de la profession, autour de trois axes : les enjeux du genre à la lumière des trajectoires professionnelles, les questions de genre au prisme des pratiques professionnelles et enfin les enjeux épistémologiques et méthodologiques des perspectives du genre pour la professionnalisation et les pratiques de la communication. Si Coulomb-Gully (2009) parle d'un « gender turn » des SIC qu'elle situe au début des années 2000, elle note néanmoins que celui-ci demeure « tout relatif». Ainsi son travail d'inventaire des revues labellisées en SIC montre que le bilan est assez maigre si on le compare à l'attention portée à la question par d'autres disciplines ou si on le compare aux travaux dans le domaine des «Media Studies » à l'international. Pourtant, SIC et études de genre ont en commun une difficile reconnaissance institutionnelle à leur naissance

1 https://cache.media.enseignementsup-recherche.gouv.fr/file/statistiques/26/1/71_1316261.pdf fiches démographiques des sections du CNU, Ministère de l'enseignement supérieur, de la recherche et de l'innovation, Août 2020. 
qui, d'ailleurs, intervient à la même période. Elles ont également en commun un ensemble de caractéristiques, dont l'attention porté au terrain (Coulomb-Gully, 2009) et l'articulation de problématiques interdisciplinaires puisque « en SIC comme pour les études de genre, il n'existe pas d'objet en soi, mais bien des manières de le constituer » (Julliard, 2009). Cette proximité nous semblait avoir été encore peu explorée, en particulier pour questionner la construction des représentations genrées et des phénomènes de pouvoir, de domination, d'invisibilisation en organisation mais aussi, plus précisément, dans le contexte de professionnalisation de la communication, et nous proposions donc d'explorer ces problématiques.

Il nous semble intéressant ici de revenir sur la fabrique de ce dossier pour mieux saisir sa composition et pour tenter d'éclairer les modalités d'appréhension du genre dans le champ des sciences de l'information et de la communication. En effet, force est de constater que la thématique reste peu explorée, en particulier dans le domaine de la communication organisationnelle. Constituer le dossier ne fut ainsi pas une entreprise aisée, le sujet ne suscitant qu'un nombre modéré de propositions - aucune ne provenant de collègues masculins. Plusieurs articles prometteurs n'ont également pas pu être menés à leur terme dans les conditions particulières de l'année 2020, marquée par une crise sanitaire mondiale, laquelle s'est traduite par une surcharge de travail qui a affecté plus sévèrement la production scientifique féminine selon un récent article de la revue Nature $^{2}$. Notons également que nous avons choisi de ne pas imposer une forme ou une autre de féminisation d'écriture, épicène ou neutre des textes. Nous avons en effet considéré que la diversité des approches retenues reflète autant de positions et de négociations de la façon dont les rapports de genre peuvent être traités dans l'écriture scientifique en sciences de la communication.

Pour autant, les articles présentés ici permettent de mieux saisir à la fois la problématique du genre en organisation par la manière dont la question est donnée à voir, mais également comment le genre participe aux modalités de la pratique professionnelle. Le premier article du dossier, signé par Emmanuelle Bruneel, analyse comment une entreprise française de services informatiques tente de valoriser, au moyen d'une campagne de communication, sa politique de " parité » et de « diversité » et, ce faisant, actualise la binarité féminin/masculin et la laisse impensée. Plutôt que de les interroger, le discours qui est donné à voir dans les supports de communication analysés dépolitise les rapports de pouvoir et de domination qui structurent les normes sociales inégalitaires de genre.

Une observation assez similaire ressort également de l'article de Sandrine Roginsky, qui porte sur la fabrique de campagnes de communication publique par des administrations fédérales belges. Elle montre que, là encore, le genre est vidé de sa dimension heuristique mais également contestataire pour être mobilisé comme critère

2 https://www.natureindex.com/news-blog/decline-women-scientist-research-publishingproduction-coronavirus-pandemic 
de catégorisation qui se substitue à la catégorie « femmes » sans prise en compte des rapports de force et, par ricochet, sans capacité d'identifier les stéréotypes de genre.

C'est dans l'univers professionnel des médias que les deux articles suivants nous amènent, d'abord en France puis au Québec. L'article de Laurence Leveneur montre comment les animatrices d'émissions de divertissement subissent une ségrégation à plusieurs facettes. Elles sont moins nombreuses, occupent des rôles énonciatifs plus limités que leurs homologues masculins, et sont souvent assignées à des coprésentations ou à des seconds rôles. Leurs trajectoires professionnelles sont aussi majoritairement assises sur un socle identitaire qui repose sur des critères de beauté physique.

On retrouve également cet élément dans l'article de Marilou St-Pierre, qui s'intéresse à l'expérience des femmes journalistes sportives au Québec. Celles-ci font face à un ensemble de stéréotypes qui s'inscrivent eux-mêmes dans une conception binaire et hétérocentrée des rapports de pouvoir, au point que le mythe de la « salope », soit l'idée répandue selon laquelle les journalistes sportives choisiraient ce métier dans le but d'entretenir des relations intimes et sexuelles avec les sportifs, s'est peu à peu implanté au Québec.

Ces quatre articles ont ainsi en commun de montrer comment, dans les différents contextes étudiés, la question du genre est neutralisée pour lui faire perdre sa dimension de conflictualité et de (re)mise en question des rapports sociaux qui s'y déploient. Ce qui n'est pas sans expliquer la circulation de stéréotypes de genre qu'on observe dans chacun des espaces professionnels explorés.

Ce dossier nous enjoint à poursuivre l'exploration et l'analyse de la professionnalisation de la communication au prisme du genre. Les articles qu'il présente témoignent en effet de situations toujours interpellantes, pour ne pas dire préoccupantes, qu'il s'agisse d'entreprises (privées et médiatiques) ou d'administrations publiques, qui devraient à cet égard retenir l'attention des chercheuses et chercheurs. La perpétuation des asymétries de genre est à ce titre un objet qui devrait interpeller les sciences de l'information et de la communication, et faire l'objet de futures recherches qui permettraient notamment d'en mieux comprendre les diverses modalités communicationnelles et les enjeux pour la pratique professionnelle en communication.

\section{Bibliographie}

Aldoory, L. et Toth, E. (2002). Gender Discrepancies in a Gendered Profession: A Developing Theory for Public Relations. Journal of Public Relations Research, 14(2), 103-126.

Bernard, F. et Loneux, C. (2014). Chercheures et recherches au féminin : point d'étape, repérage et contributions en Sciences de l'Information et de la Communication, Revue française des sciences de l'information et de la communication [Online], 4. doi : https://doi.org/10.4000/rfsic.829 
Bertini, M.-J, (2006). Un mode original d'appropriation des Cultural Studies : les Études de genre appliquées aux Sciences de linformation et de la comunication. Concepts, théories, méthodes et enjeux. MEI, 24-25, 116-124. URL : https://meiinfo.com/wp-content/uploads/revue24-25/10MEI-24-25.pdf

Caron, C. (2004). De l'appropriation inachevée du concept de genre en communication organisationnelle. Communication \& Organisation, 25. doi : https://doi. org/10.4000/communicationorganisation.2961

Coulomb-Gully, M. (2009). Les sciences de l'information et de la communication : une discipline Gender blind? Questions de communication, 15, 129-153. doi : https://doi.org/10.4000/questionsdecommunication.518

Coulomb-Gully, M. (2010). Féminin/masculin : question(s) pour les SIC. Réflexions théoriques et méthodologiques. Questions de communication, 17, 169-194. doi : https://doi.org/10.4000/questionsdecommunication.383

Daymon, Chr. et Demetrious, Kr. (eds.) (2014). Gender and PublicRelations: Critical Perspectives on Voice, Image and Identity. New York, États-Unis: Routledge

Elmer,P.(2010). Re-encountering the PR man.PRism, 7(4), 1-5.URL: https://citeseerx. ist.psu.edu/viewdoc/download?doi=10.1.1.670.760\&rep=rep1\&type $=$ pdf

Fitch, K. (2020). Wives, secretaries and bodies: Representations of women in an Australian public relations journal, 1965-1972. Dans I. Somerville, L. Edwards, Ø. Ihlen (eds.). Public Relations, Society and the Generative Power of History (pp. 61-80). New York, États-Unis: Routledge.

Fitch, K. et Third, A. (2014). Ex-journos and promo girls: Feminization and professionalization in the Australian public relations industry. Dans Chr. Daymon et Kr. Demetrious (eds.), Gender and PublicRelations: Critical Perspectives on Voice, Image and Identity (pp. 221-268). New York, États-Unis: Routledge.

Fleury, B. et Walter, J. (2009). Penser le genre en sciences de l'information et de la communication et au-delà. Questions de communication, 15, 111-127. doi : https://doi.org/10.4000/questionsdecommunication.503

Galinon-Mélénec, B. et Martin-Juchat, F. (2014). Du " genre » social au « genre » incorporé : Le « corps genré » des SIC, Revue française des sciences de l'information et de la communication [Online], 4. doi : https://doi.org/10.4000/ rfsic. 857

Grunig, L.A., Toth, E.L. et Hon, L.C. (2001). Women in Public Relations. New York, États-Unis: Guilford Press. 
Julliard, V. (2009). Pour une intégration du genre par les sciences de l'information et de la communication, Questions de communication, 16, 191-210. doi : https://doi. org/10.4000/questionsdecommunication.356

Malochet, G. (2007). La féminisation des métiers et des professions. Quand la sociologie du travail croise le genre, Sociologies Pratiques, 1(14), p. 91-99. doi : https://doi.org/10.3917/sopr.014.0091

Neveu, É. (2000). Le genre du journalisme. Des ambivalences de la féminisation d'une profession. Politix, 51(3), 179-212. doi :https://doi.org/10.3406/polix.2000.1109

Niquette, M. (2000). De la féminisation des relations publiques : un discours en rupture de l'histoire des femmes relationnistes. Recherches féministes, 13(2), 4775. doi : https://doi.org/10.7202/058096ar

Tindall, N.T.J. et Waters, R.D. (2012). Coming Out to Tell Our Stories: Using Queer Theory to Understand the Career Experiences of Gay Men in Public Relations. Journal of Public Relations Research, 24(5), 451-475.

Topic, M. et al. (2019). Women in Public Relations. A Literature Review (19822019). Euprera Report Vol. 1, № 1.

Toth, E. et Grunig, L. (1993). The Missing Story of Women in Public Relations, Journal of Public Relations Research, 5(3), 153-175. 